\title{
El desarrollo de un programa de evaluación online para la preparación de la versión informatizada del First Certificate in English
}

\author{
Ana Sevilla Pavón \\ Universidad Politècnica de València, Valéncia, España
}

(Artículo recibido el 14 de noviembre de 2011; versión final recibida el 16 de enero de 2012)

\begin{abstract}
Las exigencias actuales de la sociedad global de la comunicación han llevado a un gran número de exámenes oficiales de idiomas a adoptar el formato informatizado e incluso online, como ocurre con Cambridge First Certificate in English (FCE). El surgimiento de su versión informatizada hace que aumente la demanda de materiales preparatorios y de evaluación de calidad que tengan en cuenta las características del nuevo formato de examen, planteando ejercicios en concordancia. En este contexto se ha desarrollado el programa preparatorio y de evaluación InGenio FCE Online Tester, en el seno del grupo de investigación CAMILLE, del Departamento de Lingüística Aplicada de la Universitat Politècnica de València (UPV). El presente artículo explora las principales características del nuevo formato del FCE y describe el proceso de desarrollo del programa. Éste proporciona preparación a los candidatos mediante la práctica para el desarrollo de diferentes destrezas y competencias, y a través de la realización de simulaciones de examen.
\end{abstract}

\section{Introducción}

El examen de First Certificate in English (FCE), destinado a evaluar las destrezas de aquellos candidatos que desean certificar el estar en posesión de un nivel de inglés intermedio-alto equivalente al nivel B2 del Marco Común Europeo de Referencia para las Lenguas (Consejo de Europa, 2002) mediante la evaluación de las destrezas de comprensión y expresión, tanto escritas como orales, venía realizándose desde hacía varias décadas en el formato tradicional impreso. No obstante, siguiendo los pasos de otros prestigiosos exámenes de oficiales de alto impacto social (tales como el TOEFL) y, con el objetivo de proporcionar la máxima eficiencia y efectividad - factores clave en la sociedad actual de la comunicación-, este año el FCE presenta la novedad de poder realizarse a través del ordenador. Así, en enero de 2010 se comunicó en la página Web oficial de University of Cambridge ESOL Examinations la incorporación de la nueva prueba de FCE asistida por ordenador, la así llamada Computer-based First Certificate in English Examination (CBFCE). 
El surgimiento de la nueva versión informatizada del FCE, denominada Computer-based First Certificate in English Examination (CBFCE), hace que aumente la demanda, por parte de profesores y estudiantes, de materiales preparatorios en cuyo desarrollo, además de cuidarse al máximo la calidad, se tengan en cuenta las características del nuevo formato de examen y se planteen ejercicios en concordancia con dicho formato. El FCE Online Tester surge como respuesta del grupo de investigación CAMILLE, de la Universitat Politècnica de València (UPV), ante este hecho. El presente artículo describe el proceso de desarrollo de dicho programa de preparación y evaluación, implementado mediante el sistema InGenio, al tiempo que presenta las ventajas del nuevo formato informatizado del examen y describe los beneficios de la utilización de dicho programa a la hora de preparar el FCE. El FCE Online Tester fue desarrollado con la idea de suplir la anterior carencia de materiales preparatorios y de evaluación con estas características. Su utilización permite a los candidatos familiarizarse con el nuevo formato de examen, al tiempo que realizan ejercicios preparatorios y simulaciones online de examen que fomentan la práctica y el desarrollo de destrezas similares a las evaluadas por las diferentes secciones de que consta el FCE. Dado que el conocimiento lingüístico es complejamente dinámico y moldeado en contexto (Hall, Cheng, y Carlson, 2006), en el desarrollo del Tester se ha tenido en cuenta que el uso de las Tecnologías de la Información y de las Comunicaciones (TIC) está cada vez más extendido, tanto en el ámbito privado como en el académico y profesional, quedando patente la predominancia de la lengua inglesa. La supremacía del inglés y la estrecha relación entre esta lengua y las TIC apuntan en dirección a la integración de las TIC en el proceso de enseñanza / aprendizaje del inglés, pues las TIC expanden el papel del inglés en el mundo y tienen un fuerte impacto en el contexto y en la manera en que se enseñan las lenguas (Warschauer, 2006).

Por otro lado, el punto de partida del diseño del Tester ha sido el aprendizaje centrado en el estudiante. Además, se ha adoptado un enfoque sociocultural en que el estudiante pasa a ser un agente activo y responsable de su propio aprendizaje, con lo que uno de los principales objetivos del diseño y del análisis es "to build awareness of and fluency in the kinds of instructional guidance and responsive assistance that maximize learner-centered opportunities for their generative language use” (Meskill y Sadykova, 2011:201). La parte correspondiente a la preparación del examen se realiza a través de ejercicios de tipologías semejantes a los del examen real, mientras que la evaluación y autoevaluación vienen de la mano de las simulaciones de examen. Gracias a la 
realización de las simulaciones, se le permite al candidato no sólo conocer el formato del examen informatizado, sino también predecir los resultados que obtendría en la prueba real e incluso detectar áreas concretas de dificultad. El que dichas áreas de dificultad le sean indicadas al candidato antes de que el mismo se enfrente al examen real es un elemento clave, ya que proporciona al candidato la oportunidad de tratar de atajar dichas dificultades de cara a obtener mejores resultados en el examen (Sevilla Pavón y Martínez Sáez, 2010).

\section{Características generales del CBFCE}

La versión informatizada del FCE no introduce cambios en cuanto al contenido del examen, ni tampoco en lo relacionado con: las partes de que consta el examen, la tipología de ejercicios y la puntuación que se le asigna a cada una de las partes en que se divide. El FCE es una prueba oficial perteneciente al University of Cambridge Local Examinations Syndicate (UCLES), ampliamente reconocida a nivel internacional, destinada a evaluar las destrezas de aquellos candidatos que desean certificar la posesión de un nivel de inglés intermedio-alto, equivalente al nivel B2 del Marco común europeo de referencia para las lenguas (MCERL) (Consejo de Europa, 2002). Se trata de un examen que evalúa las cuatro destrezas básicas, es decir, las destrezas de comprensión y expresión, tanto escritas como orales. La evaluación de dichas destrezas en la prueba informatizada mantiene la estructura del examen impreso, distribuyéndose en cinco secciones o "Papers", correspondientes a la expresión oral y escrita, la comprensión oral y escrita, y el uso del inglés (esto es, gramática y vocabulario).

La finalidad de los diferentes exámenes de Cambridge ESOL, de acuerdo con la University of Cambridge ESOL Examinations (ver UCLES; http://www.cambridgeesol.org/index.html), es proporcionar a estudiantes y profesores de idiomas, en una variedad de situaciones, el acceso a una amplia gama de exámenes internacionales de gran calidad, tests y diplomas, que les ayuden a lograr sus metas personales y que repercutan favorablemente en su experiencia de aprendizaje y desarrollo profesional.

El FCE certifica que quien está en su posesión posee el suficiente dominio de la lengua inglesa para ocupar puestos administrativos o de gerencia en diversos sectores empresariales, y los candidatos que aprueban el examen reciben, además del certificado concedido por UCLES, una relación de todos los resultados obtenidos en las cinco pruebas de las que consta el examen. El nivel de competencia que acredita el FCE 
supone que el candidato es un usuario independiente, capaz de captar el mensaje esencial de textos con temas de carácter concreto y abstracto y participar activamente en una conversación técnica dentro de su campo de especialización, comunicarse con suficiente espontaneidad y fluidez con hablantes nativos sin que la conversación involucre un esfuerzo especial por parte de ninguno de los interlocutores, expresarse de manera clara y matizada sobre temas diversos y comentar noticias de actualidad, examinando las ventajas y desventajas de situaciones concretas (ver UCLES, http://www.cambridgeesol.org/index.html).

La aparición de dos nuevas pruebas de inglés asistidas por ordenador de Cambridge fue anunciada en enero de 2010 a través de la página Web oficial de UCLES. Dichas pruebas informatizadas son FCE y Certificate in Advanced English (CAE). Éstas se suman a las pruebas de Cambridge ESOL que ya estaban disponibles en dicho formato, ocupando el quinto lugar en cuanto a nivel, tal y como muestra la siguiente tabla en que figuran los diferentes exámenes oficiales, situándose en la parte inferior el nivel más bajo, y en la parte superior el nivel más alto. La siguiente tabla muestra, además, las equivalencias entre los niveles de los exámenes oficiales de Cambridge y el MCERL.

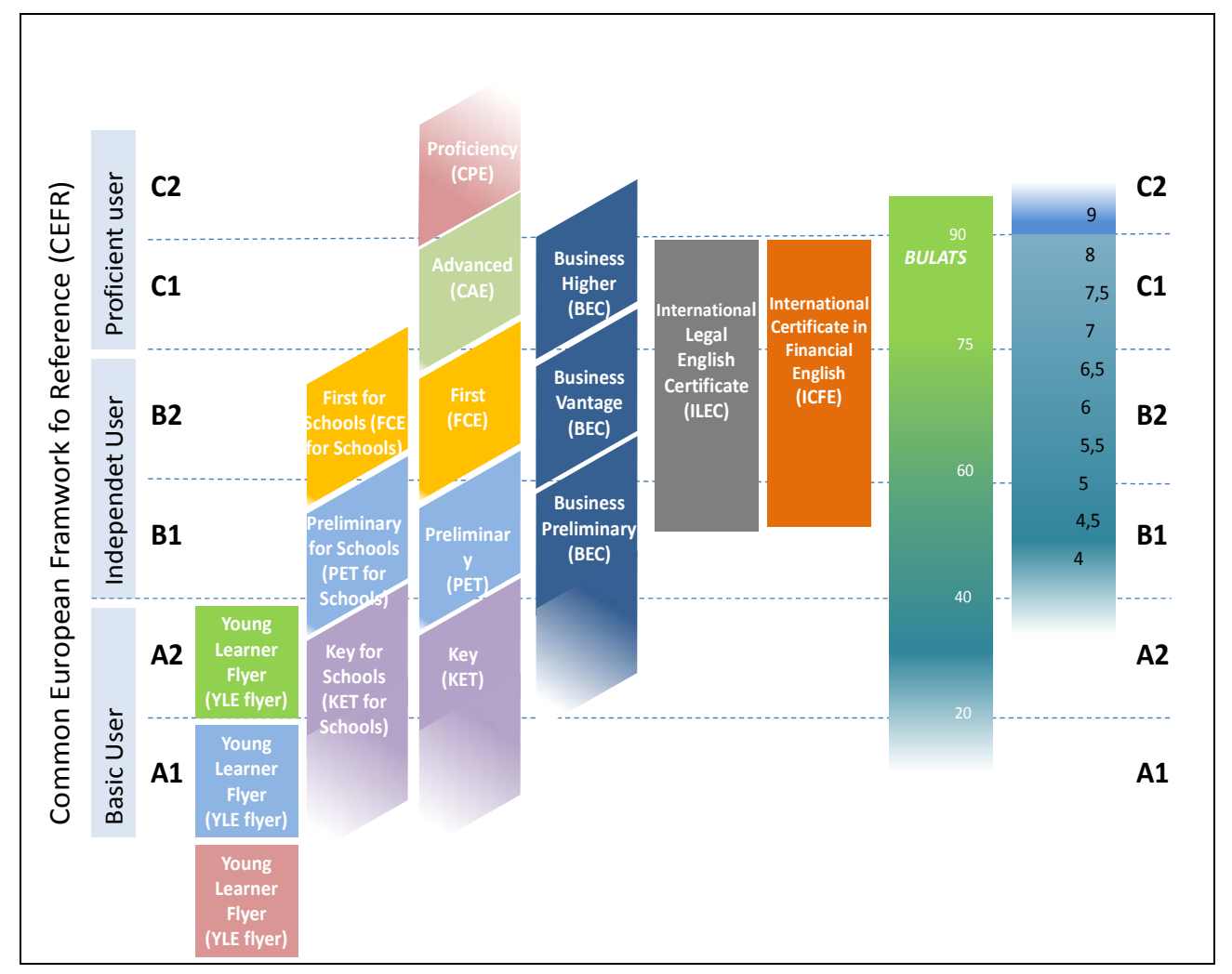

Figura 1. Diagrama de equivalencias entre los niveles de los exámenes oficiales de Cambridge y el MCERL, basado en el diagrama de Cambridge ESOL 
Debido al gran número de ventajas ofrecidas por el formato informatizado, y en un intento de atender a las necesidades en materia comunicativa de la actual sociedad basada en la comunicación intercultural y plurilingüe (con la predominancia de la lengua inglesa), desde hacía varios años se venía estudiando la posibilidad de informatizar los diferentes exámenes de Cambridge. Así, en 2007, en la versión electrónica del Boletín de Noticias $n^{\circ} 24$ de Cambridge, se anunciaban los planes de introducir nuevas versiones informatizadas de algunos de sus exámenes, y se adelantaba que las pruebas piloto realizadas con los exámenes en el nuevo formato habían sido todo un éxito, pues los candidatos participantes en dichas pruebas afirmaron considerar que los ordenadores eran una herramienta muy práctica y útil para la realización de tipo de exámenes. Además, las pruebas piloto permitieron concluir que sería posible gestionar con éxito la seguridad en este tipo de exámenes. También se observó que los candidatos se mostraban satisfechos respecto al uso de auriculares con volumen regulable para la realización de la parte de comprensión oral de los exámenes oficiales realizados; al tiempo que valoraban positivamente el poder volver a los ejercicios en cualquier momento durante la realización del examen para así poder introducir cuantas modificaciones consideraran necesarias.

Además de las ventajas señaladas por los participantes en las pruebas piloto, existen una serie de ventajas adicionales en relación a la informatización de las pruebas del Cambridge, entre las cuales se incluyen:

- la posibilidad de acceder más rápidamente a los resultados, estando las calificaciones obtenidas disponibles en la Web tan solo tres semanas después del test;

- el que un gran número de estudiantes pueda realizar el examen de manera simultánea y más cómoda;

- la posibilidad de modificar y corregir las respuestas en la pantalla durante la realización del examen;

- el uso de auriculares en las partes de comprensión oral, que no sólo ofrece la posibilidad de ajustar el volumen a las necesidades de cada alumno, sino que, además, proporciona una mayor calidad de sonido, eliminando las posibles interferencias externas;

- y la incorporación de funciones tales como el control del tiempo online, así como la función de ayuda en caso de dudas, cuya consulta está disponible en cualquier momento del examen, sin necesidad de intervención externa. 


\section{Contenidos y estructura del programa}

Por ser el Tester un programa dirigido a la preparación del examen del FCE, los contenidos, el nivel y la estructura del mismo han sido en gran medida determinados por la estructura y por los contenidos del propio examen de inglés. Así, los ejercicios propuestos corresponden a las destrezas evaluadas en el FCE: comprensión lectora, expresión escrita, comprensión auditiva y expresión oral, incluyéndose también una parte dedicada al "uso de la lengua”, la cual se corresponde con la parte de gramática y de vocabulario.

En la división de cada unidad en cinco apartados, correspondientes a las cinco secciones de que consta el FCE, se parte de la base de que una mayor similitud con el examen en cuanto a contenidos y estructura podrá mejorar los resultados de los candidatos, que se sentirán más cómodos a la hora de enfrentarse al examen real, por haber podido tanto familiarizarse con su formato, como practicar destrezas similares a las que serán evaluadas, a través de ejercicios semejantes a los propuestos en el examen del FCE. Con el fin de proporcionar información detallada sobre la evolución de los estudiantes, el programa de evaluación incluye un apartado accesible a través de la Web que permite al profesor (tutor) y al estudiante supervisar el trabajo realizado y comprobar la evolución del aprendizaje, a través de la plantilla correspondiente al “informe de evaluación del estudiante”, que se muestra a continuación: 


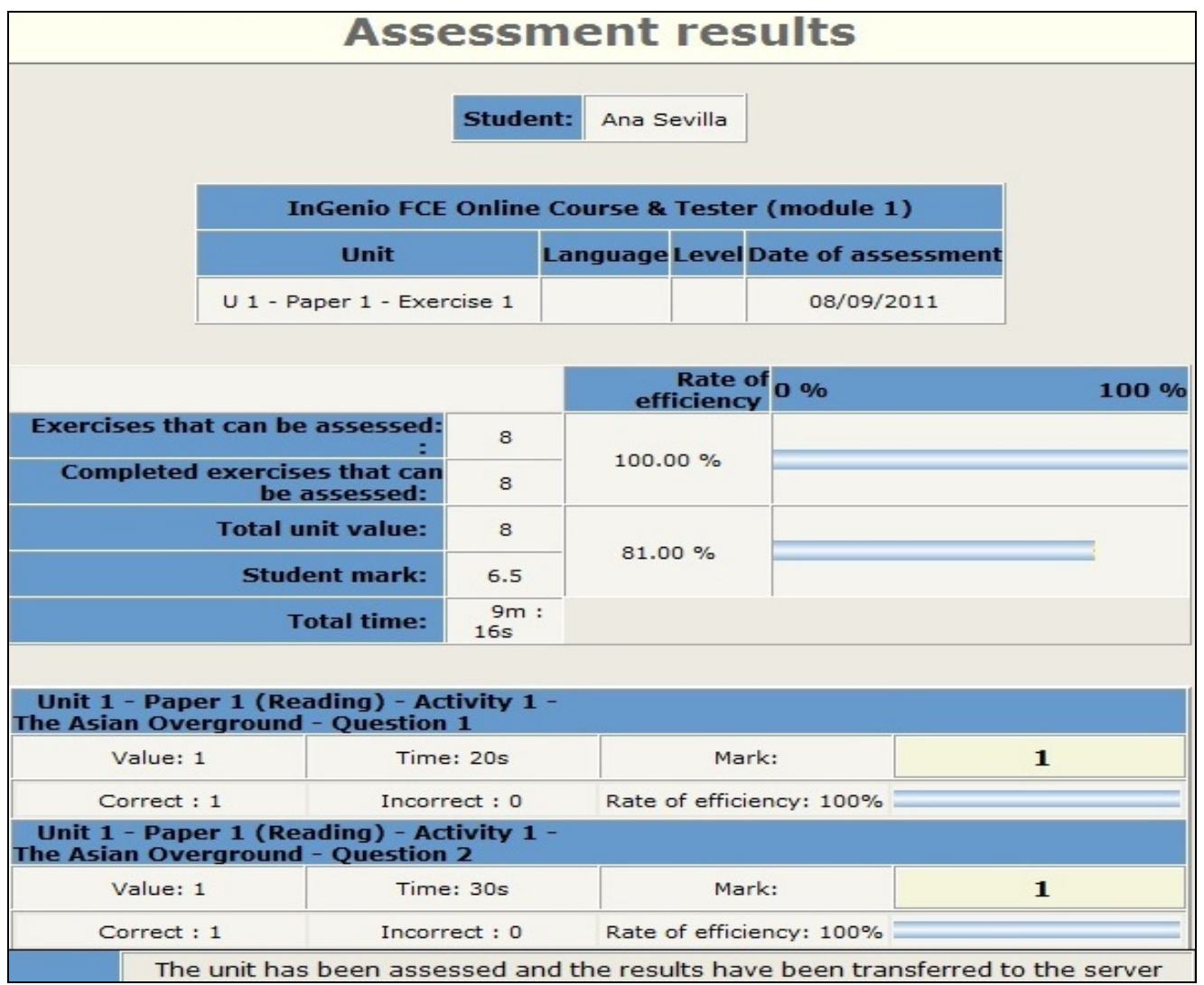

Figura 2. Informe de evaluación del estudiante en InGenio

En cuanto a los contenidos, en el caso del curso que se usa en combinación con el Tester, denominado InGenio FCE Online Course, éste se centra en contenidos teóricos y prácticos, e incluye asimismo actividades destinadas a la práctica y la familiarización con el examen, respetando en muchos casos la tipología de ejercicios incluidos en el examen oficial. Al mismo tiempo, también incluye ejercicios complementarios centrados en la explicación y la práctica de aspectos específicos -gramaticales, léxicos, fonéticos, etc., con el fin de fomentar la práctica de aquellos elementos lingüísticos que más dificultades planteen. Por otro lado, el curso incluye una serie de contenidos técnicos específicos de cada una de las licenciaturas de la UPV, centrados en las disciplinas de Lenguas para fines específicos (LFE) y de aprendizaje integrado de contenidos y de lengua (AICLE).

En lo relativo al diseño del Tester, éste se ha efectuado mediante la creación de una base de datos a través de la cual es posible acceder a corpus de textos, así como a ejercicios previamente introducidos, todos ellos actualizados de manera periódica, y susceptibles de ser usados en diversas combinaciones, a petición del estudiante o del tutor. De este modo, es posible realizar a partir de ellos simulaciones de examen que emulan el entorno del FCE en términos de formato, tipología de ejercicios y tiempo 
disponible para la realización del mismo. Asimismo, permite la práctica de las diferentes destrezas requeridas para la superación del examen (Gimeno Sanz, Martínez Sáez, Sevilla Pavón, y de Siqueira Rocha, 2011).

La práctica y preparación se basan principalmente, según lo anteriormente expuesto, en la realización de ejercicios online de nivel B2, la gran mayoría de los cuáles posee una tipología semejante a la de los propuestos en el examen del FCE. Asimismo, se incluyen actividades y ejercicios de tipologías complementarias, destinados a trabajar aspectos lingüísticos concretos y contenidos específicos. Además, dicha práctica y preparación son facilitadas por las múltiples utilidades de la plataforma InGenio: enlaces externos, explicaciones concretas que emergen automáticamente, creación de diccionarios específicos, etc. Por su parte, la realización de simulaciones del examen del FCE tiene como objetivo primordial el permitir al candidato predecir sus posibles resultados en la prueba y conocer si su preparación para el examen es la adecuada, así como localizar aquellas áreas lingüísticas que presentan mayores dificultades, para así poder mejorar antes de la realización de la prueba, de cara a obtener mejores resultados en la misma. Si bien la apariencia gráfica no es idéntica a la del examen oficial, en las simulaciones sí se respetan las tipologías de ejercicios incluidas en el examen. Esto hace que dicho programa pueda resultar útil no sólo de cara a la práctica y evaluación de contenidos y destrezas, tomando como modelo la estructura del examen oficial; sino también en lo que respecta a la adaptación de los estudiantes y a su familiarización con el formato informatizado, el cual viene siendo utilizado en un número creciente de centros examinadores desde principios de 2010.

Todo lo señalado anteriormente es posible mediante el uso de las plantillas predeterminadas de las que InGenio dispone, las cuales son adaptadas previamente a los requisitos, formato y características concretas del examen del FCE. Estas plantillas “aseguran variedad, coherencia, consistencia y riqueza” (Gimeno Sanz, 2006:5) y se caracterizan por "ser lo más abiertas y flexibles posible, con el fin de apoyar el diseño de actividades creativas e imaginativas, en lugar de restringir y confinar los contenidos durante el proceso creativo” (Gimeno Sanz, 2006:5). Además, dichas plantillas permiten la creación de diversos tipos de ejercicios, y son susceptibles de ser adaptadas o modificadas por los autores de contenidos a los que previamente se les haya concedido los permisos oportunos para ello. Las principales plantillas corresponden a ejercicios de observación, ejercicios de vídeo, ejercicios de opción múltiple con solución única en un menú desplegable, ejercicios de opción múltiple con solución única de entre varias 
opciones, ejercicios de opción múltiple con solución múltiple, ejercicios de rellenar huecos, ejercicios de ordenación de palabras o frases, ejercicios pasivos de respuesta única a través de una imagen clicable, ejercicios activos de respuesta múltiple a través de una imagen clicable, ejercicios de texto libre, dictado, ejercicios de asociación y pasatiempos. A continuación se muestran dos ejemplos de ejercicios creados a partir de plantillas de InGenio:

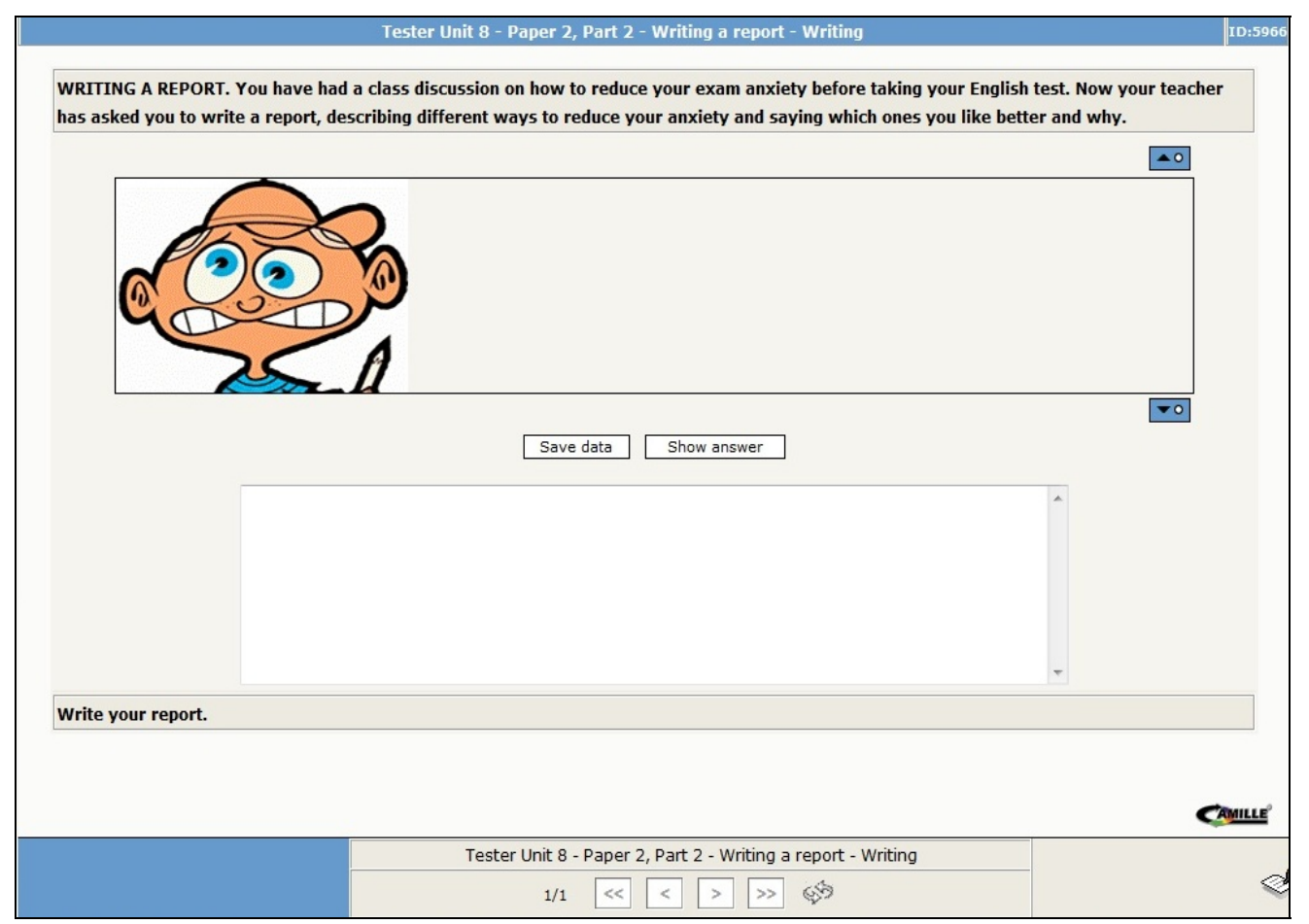

Figura 3. Ejemplo de ejercicio de expresión escrita, creado con la plantilla de “redacción” de InGenio

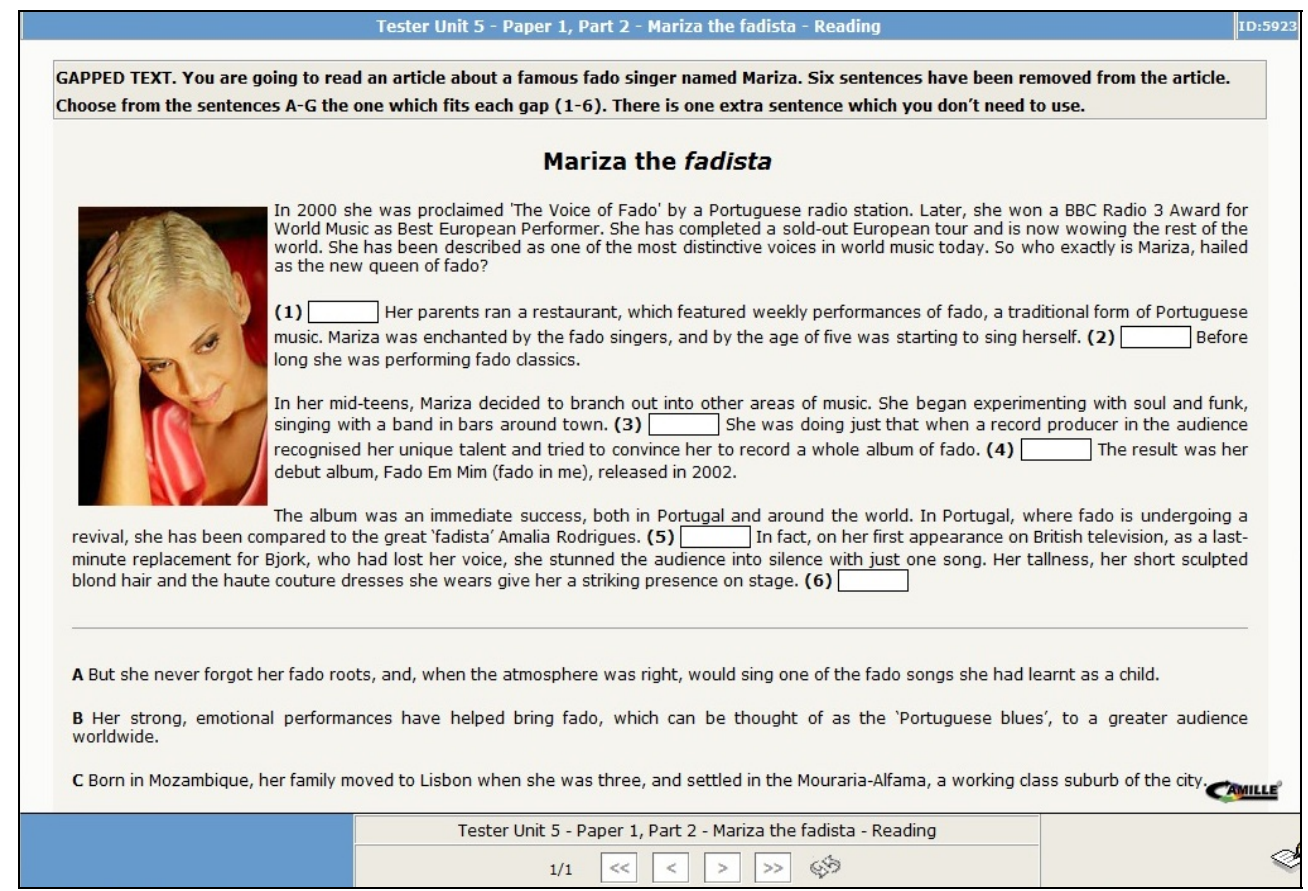

Figura 4. Ejemplo de ejercicio de comprensión escrita creado con la plantilla de "rellenar huecos” de InGenio 
El programa presenta dos posibilidades de uso, puesto que es susceptible de ser usado tanto en conjunción con el FCE Online Course, como a modo de recurso independiente. En el primer caso, en que el programa es usado al tiempo que se usa también el FCE Online Course, los contenidos del programa se organizan en dos tipos de unidades, incluyendo contenidos de evaluación general, el primero; y de evaluación y preparación específica (con una cantidad de contenidos menor), el segundo tipo.

El primer tipo de unidades, más amplio y general, consiste en unidades compuestas fundamentalmente por ejercicios de práctica y de evaluación de las diferentes destrezas, a través de los cuales se adquirirán y evaluarán conocimientos y habilidades de nivel B2, siendo éstos tanto los que figuran entre los descriptores del MCERL como los evaluados en exámenes oficiales de dicho nivel, como el FCE. Tras realizar los ejercicios correspondientes, los estudiantes reciben el feedback correspondiente, tanto de tipo general como específico, y son referidos a los contenidos teóricos apropiados en cada caso, en función del área en que cada uno de los estudiantes presente dudas o dificultades. El segundo tipo de unidades es, como decimos, más reducido y más específico, pues consiste en una serie de ejercicios, ejemplos y modelos centrados cada uno de ellos en una destreza específica.

Los materiales incluyen, además de los diferentes contenidos, consejos de estudio y de preparación de exámenes oficiales de nivel B2 tales como el FCE, así como referencias y enlaces a los materiales de consulta pertinentes. Todos estos recursos adicionales, en formato impreso y electrónico, son escogidos de acuerdo con criterios de calidad y utilidad en cuanto a la preparación y práctica específica de cada una de las destrezas. Además, son adecuados tanto a la hora de realizar dicha práctica y preparación de manera separada, como para conectar y relacionar una destreza en concreto con el resto de destrezas y habilidades que se incluyen en los descriptores del nivel B2.

Ambos tipos, general y específico, se organizan en el Tester del siguiente modo: El programa está compuesto por dos módulos formados por un total de 16 unidades de evaluación y de preparación, intercalándose el primero tipo de unidades con el segundo, al tiempo que se les proporcionan a los estudiantes consejos de examen y estrategias de estudio, así como un amplio número de ejercicios de práctica, y de ejemplos y modelos. El primer módulo en que se divide el Tester abarca desde la primera unidad hasta la octava, mientras que las ocho unidades restantes corresponden al segundo módulo 
programa. Las siguientes tablas muestran el esquema organizativo de las unidades de que consta cada uno de los módulos del Tester, proporcionando visión general de los contenidos y de las destrezas tratadas en cada una de las unidades:

Tabla 1. Esquema general de contenidos del módulo 1 de InGenio FCE Online Tester

\begin{tabular}{|c|c|c|c|c|}
\hline \multicolumn{5}{|c|}{ Unidades y Contenidos del Tester - Módulo 1} \\
\hline Unidad & Título & Contenidos & Destrezas & Temas generales \\
\hline 1 & $\begin{array}{c}\text { You are what } \\
\text { you eat }\end{array}$ & $\begin{array}{l}\text { Contenidos teóricos en } \\
\text { diferentes formatos, } \\
\text { ejercicios, feedback, } \\
\text { descripción del examen } \\
\text { (Paper 1). }\end{array}$ & R-W-UoE-L-S & $\begin{array}{l}\text { Alimentación y } \\
\text { salud, actividades de } \\
\text { ocio y tiempo libre. }\end{array}$ \\
\hline 2 & $\begin{array}{l}\text { It's all about } \\
\text { business }\end{array}$ & $\begin{array}{l}\text { Contenidos teóricos en } \\
\text { diferentes formatos, } \\
\text { ejercicios, feedback, } \\
\text { descripción del examen } \\
\text { (Paper 2). }\end{array}$ & R-W-UoE-L-S & $\begin{array}{l}\text { Consumismo, } \\
\text { economía, ética en } \\
\text { los negocios, el } \\
\text { mundo laboral. }\end{array}$ \\
\hline 3 & $\begin{array}{c}\text { Move it or lose } \\
\text { it }\end{array}$ & $\begin{array}{l}\text { Contenidos teóricos en } \\
\text { diferentes formatos, } \\
\text { ejemplos, modelos, } \\
\text { ejercicios, consejos } \\
\text { examen, estrategias de } \\
\text { examen, referencias a otros } \\
\text { materiales de utilidad. }\end{array}$ & R-W-UoE-L- S & $\begin{array}{l}\text { Las destrezas de } \\
\text { comprensión } \\
\text { lectora: teoría y } \\
\text { práctica. } \\
\text { Salud, estilo de vida } \\
\text { activo y saludable. }\end{array}$ \\
\hline 4 & $\begin{array}{l}\text { Save the } \\
\text { Planet }\end{array}$ & $\begin{array}{l}\text { Contenidos teóricos en } \\
\text { diferentes formatos, } \\
\text { ejercicios, feedback, } \\
\text { descripción del examen } \\
\text { (Paper 3). } \\
\end{array}$ & R-W-UoE-L-S & $\begin{array}{c}\text { Ecología, } \\
\text { sostenibilidad, } \\
\text { naturaleza, deportes } \\
\text { al aire libre. }\end{array}$ \\
\hline 5 & $\begin{array}{l}\text { Let the beat } \\
\text { rock! }\end{array}$ & $\begin{array}{l}\text { Contenidos teóricos en } \\
\text { diferentes formatos, } \\
\text { ejercicios, feedback, } \\
\text { descripción del examen } \\
\text { (Paper 4). }\end{array}$ & R-W-UoE-L-S & $\begin{array}{l}\text { Música, baile, } \\
\text { diversión y } \\
\text { expresión a través } \\
\text { de la música, } \\
\text { industria musical. }\end{array}$ \\
\hline 6 & $\begin{array}{l}\text { It's a small } \\
\text { world }\end{array}$ & $\begin{array}{l}\text { Contenidos teóricos en } \\
\text { diferentes formatos, } \\
\text { ejemplos, modelos, } \\
\text { ejercicios centrados } \\
\text { principalmente en la } \\
\text { expresión escrita, consejos } \\
\text { de examen / estrategias, } \\
\text { referencias a otros } \\
\text { materiales de utilidad. }\end{array}$ & R-W-UoE-L- S & $\begin{array}{l}\text { Tradiciones y } \\
\text { celebraciones, } \\
\text { culturas del mundo } \\
\text { en general y de } \\
\text { diferentes países de } \\
\text { habla inglesa. }\end{array}$ \\
\hline 7 & $\begin{array}{l}\text { I need a } \\
\text { holiday! }\end{array}$ & $\begin{array}{l}\text { Contenidos teóricos en } \\
\text { diferentes formatos, } \\
\text { ejercicios, feedback, } \\
\text { descripción del examen } \\
\text { (Paper 5). }\end{array}$ & R-W-UoE-L- S & $\begin{array}{l}\text { Viajes, vacaciones, } \\
\text { tiempo libre, } \\
\text { turismo ético y } \\
\text { ecológico. }\end{array}$ \\
\hline 8 & Hi-tech & $\begin{array}{l}\text { Contenidos teóricos en } \\
\text { diferentes formatos, } \\
\text { ejercicios, feedback, } \\
\text { descripción examen (modo } \\
\text { de puntuación). }\end{array}$ & R-W-UoE-L- S & $\begin{array}{l}\text { Tecnología, lo } \\
\text { antiguo y lo } \\
\text { moderno, aspectos } \\
\text { técnicos de } \\
\text { especialidad, } \\
\text { medios de } \\
\text { transporte. } \\
\end{array}$ \\
\hline
\end{tabular}


Tabla 2. Esquema general de contenidos del módulo 2 de InGenio FCE Online Tester

\begin{tabular}{|c|c|c|c|c|}
\hline \multicolumn{5}{|c|}{ Unidades y Contenidos del Tester - Módulo 2} \\
\hline Unidad & Título & Contenidos & Destrezas & Temas generales \\
\hline 9 & $\begin{array}{c}\text { The Social } \\
\text { Web }\end{array}$ & $\begin{array}{l}\text { Contenidos teóricos en } \\
\text { diferentes formatos, } \\
\text { ejemplos, ejercicios } \\
\text { centrados principalmente } \\
\text { en el uso del inglés } \\
\text { (gramática y vocabulario), } \\
\text { consejos de examen/ } \\
\text { estrategias, referencias a } \\
\text { otros materiales, } \\
\text { descripción del examen } \\
\text { (Paper 1). }\end{array}$ & $\begin{array}{c}\text { R-W-UoE-L- } \\
\text { S }\end{array}$ & $\begin{array}{l}\text { La WWW, internet, } \\
\text { las TIC, los nuevos } \\
\text { modos de } \\
\text { comunicación y los } \\
\text { cambios sociales } \\
\text { relacionados. }\end{array}$ \\
\hline 10 & Life in colour & $\begin{array}{l}\text { Contenidos teóricos en } \\
\text { diferentes formatos, } \\
\text { ejemplos, ejercicios, } \\
\text { feedback. }\end{array}$ & $\begin{array}{c}\text { R-W-UoE-L- } \\
\text { S }\end{array}$ & $\begin{array}{c}\text { Arte, } \\
\text { manifestaciones } \\
\text { artísticas del } \\
\text { presente y del } \\
\text { pasado. }\end{array}$ \\
\hline 11 & A great story & $\begin{array}{l}\text { Ejercicios, feedback, } \\
\text { descripción examen } \\
\text { examen (Paper 2). }\end{array}$ & $\begin{array}{c}\text { R-W-UoE-L- } \\
\text { S }\end{array}$ & $\begin{array}{l}\text { Películas, libros, } \\
\text { ficción y realidad, } \\
\text { fenómenos } \\
\text { paranormales. }\end{array}$ \\
\hline 12 & $\begin{array}{l}\text { Mind your } \\
\text { language }\end{array}$ & $\begin{array}{c}\text { Contenidos teóricos en } \\
\text { diferentes formatos, } \\
\text { ejemplos, modelos, } \\
\text { ejercicios comprensión } \\
\text { auditiva. } \\
\text { Consejos de examen / } \\
\text { estrategias, descripción del } \\
\text { examen (Paper 3). } \\
\end{array}$ & $\begin{array}{c}\text { R-W-UoE-L- } \\
\text { S }\end{array}$ & $\begin{array}{l}\text { Lenguaje y } \\
\text { comunicación, el } \\
\text { inglés como lengua } \\
\text { internacional, } \\
\text { world englishes. }\end{array}$ \\
\hline 13 & $\begin{array}{l}\text { Nothing but } \\
\text { the truth }\end{array}$ & $\begin{array}{l}\text { Ejercicios, feedback, } \\
\text { descripción examen } \\
\text { examen (Paper 4). }\end{array}$ & $\begin{array}{c}\text { R-W-UoE-L- } \\
\text { S }\end{array}$ & $\begin{array}{l}\text { Delitos, cuestiones } \\
\text { de seguridad } \\
\text { personal e } \\
\text { informática, } \\
\text { prevención de } \\
\text { riesgos y de delitos. }\end{array}$ \\
\hline 14 & $\begin{array}{l}\text { Working for a } \\
\text { living }\end{array}$ & $\begin{array}{l}\text { Contenidos teóricos en } \\
\text { diferentes formatos, } \\
\text { ejercicios, feedback, } \\
\text { descripción del examen } \\
\text { (Paper 5). }\end{array}$ & $\begin{array}{c}\text { R-W-UoE-L- } \\
\text { S }\end{array}$ & $\begin{array}{l}\text { Estudios, trabajo y } \\
\text { profesiones, futuro } \\
\text { profesional. }\end{array}$ \\
\hline 15 & We are family & $\begin{array}{c}\text { Contenidos teóricos en } \\
\text { diferentes formatos, } \\
\text { ejemplos, modelos, } \\
\text { ejercicios centrados } \\
\text { principalmente en la } \\
\text { práctica de la expresión } \\
\text { oral, consejos de examen / } \\
\text { estrategias, referencias a } \\
\text { otros materiales. } \\
\end{array}$ & $\begin{array}{c}\text { R-W-UoE-L- } \\
\text { S }\end{array}$ & $\begin{array}{c}\text { Relaciones } \\
\text { familiares, amigos } \\
\text { y compañeros } \\
\text { sentimentales, } \\
\text { animales y } \\
\text { mascotas. }\end{array}$ \\
\hline 16 & Test yourself! & $\begin{array}{l}\text { Simulación de un examen } \\
\text { oficial, con ejercicios } \\
\text { destinados a la evaluación } \\
\text { de las diferentes destrezas } \\
\text { escritas y orales. }\end{array}$ & $\begin{array}{c}\text { R-W-UoE-L- } \\
\text { S }\end{array}$ & $\begin{array}{l}\text { Todos los temas de } \\
\text { las unidades } \\
\text { anteriores. }\end{array}$ \\
\hline
\end{tabular}




\section{Objetivos y funciones del programa}

Las funciones principales de este programa son, por un lado, proporcionar una preparación adecuada para el nivel B2 del MCERL; y, por otro lado, permitir la realización de pruebas de evaluación y autoevaluación online de nivel B2, de acuerdo con las exigencias del examen FCE y, más concretamente, de su versión informatizada CBFCE. El programa es susceptible de ser utilizado conjuntamente con el curso online de preparación para dicho examen, FCE Online Tester, en cuyo caso ambos materiales serían empleados de manera complementaria. Este programa se ha servido de los últimos avances en ALAO, siguiendo la línea de creación de recursos, objetos de aprendizaje y materiales educativos multimedia (MEM) para la enseñanza, el aprendizaje y la evaluación de lenguas extranjeras, con especial atención a dos modalidades de aprendizaje: el autoaprendizaje y el aprendizaje autónomo.

El programa FCE Online Tester está destinado, como decimos, a la preparación y evaluación / autoevaluación en línea de los estudiantes que se estén preparando para una prueba de nivel B2 en general y, en particular, de aquellos que se estén preparando para realizar el examen oficial FCE. En su faceta de herramienta destinada a la evaluación y a la autoevaluación, el Tester posee el doble objetivo de, por un lado, preparar a los estudiantes para una prueba oficial tal como el FCE, que corresponde al nivel B2 exigido para los licenciados en todas las carreras técnicas de diversas universidades donde el Plan Bolonia ha entrado en vigor, como la Universidad Politécnica de Valencia (UPV); y, por otro lado, poner a los alumnos en una situación parecida a la que vivirán a la hora de afrontar el examen, y así hacerles consciente de cuáles son los aspectos concretos que presentan mayores dificultades para cada uno de los candidatos, dándoles la posibilidad de trabajar dichos aspectos antes de la realización de la prueba para, de este modo, atajar las dificultades y mejorar los resultados. Dichos objetivos se persiguen desde una perspectiva sociocultural y centrada en el estudiante, según la cual la metodología empleada fomenta la autonomía de los estudiantes, al convertirles en elementos activos y responsables de su propio proceso de aprendizaje. Además, se pretende dar a los estudiantes una mayor seguridad a la hora de afrontar la prueba del FCE o cualquier otro tipo de examen oficial, haciendo que sean conscientes de sus posibilidades y limitaciones, y permitiendo que sepan de antemano, de un modo aproximado, cuáles serán sus resultados en dicha prueba. Esta posibilidad 
de predicción de los resultados podría influir muy positivamente en el desempeño de los estudiantes en el examen, al reducir sus niveles de ansiedad.

Este programa puede ser usado asimismo para generar elementos de evaluación en línea, proporcionado una valiosa información disponible en todo momento para el tutor, la cual le permitiría observar y evaluar de forma detallada e individualizada el progreso de cada uno de los alumnos. Este último aspecto es de gran utilidad en los casos en que dicho programa es utilizado no sólo para la preparación del examen del FCE, sino también como herramienta didáctica y de evaluación en sí misma. Por otro lado, a través de un breve test de nivel que se incluyen en el programa, el estudiante puede saber el nivel de que parte antes de comenzar a usar el Tester, siéndole posible posteriormente compararlo con el nivel alcanzado al finalizarlo. Una vez completados todas las actividades y ejercicios propuestos, el candidato podrá proceder a realizar una serie de simulaciones de examen generadas por el propio programa. Dichas simulaciones de examen pueden ser generadas a petición del tutor o del usuario una vez realizado el curso. El amplio número de corpus de textos y de ejercicios introducidos en la base de datos permiten que las simulaciones sean muy variadas, gracias al extenso número de combinaciones aleatorias posibles de ejercicios y actividades.

\section{Conclusión}

La creación de este programa de evaluación online, diseñado mediante el sistema InGenio, es un buen ejemplo de cómo las TIC pueden ser integradas en el proceso de enseñanza y aprendizaje del inglés, aprovechando las múltiples ventajas que los constantes avances tecnológicos ofrecen en la actualidad. Este artículo se ha centrado en el proceso de desarrollo del programa preparatorio y de evaluación FCE Online Tester, recurso del sistema InGenio destinado a evaluación y a la preparación de exámenes de nivel B2 tales como el FCE, tanto en su versión impresa como, más especialmente, su versión informatizada o CBFCE.

En el desarrollo del Tester se ha prestado especial atención al doble papel que dicho programa desempeña, tanto como componente del sistema InGenio, a nivel micro; como dentro del contexto más amplio de la UPV, cuyos estudiantes tienen, en su inmensa mayoría, un perfil científico-técnico que determina en gran medida sus necesidades lingüísticas específicas.

El Tester es el resultado de combinar las TIC y los esfuerzos de implementación tecnológica con la publicación y utilización de recursos online destinados a la enseñanza 
y a la evaluación de los conocimientos del inglés como lengua extranjera. El Tester pretende suplir la anterior carencia de materiales preparatorios y de evaluación con las características descritas a lo largo del presente artículo, que atiendan, en lo que a materiales didácticos se refiere, las necesidades de estudiantes que quieran certificar el estar en posesión de un nivel intermedio-alto de inglés, esto es, de un nivel B2, de acuerdo con el MCERL. Dicho programa ha sido desarrollado en un momento histórico en que la tecnología está pasando de ser una alternativa más a convertirse, en cada vez más lugares (también en el ámbito de la educación y de la enseñanza de lenguas), en una realidad a la que no podemos ni debemos seguir dando la espalda.

\section{Referencias Bibliográfícas}

Cambridge First, Newsletter 24, http://www.cambridgeesol.org/what-wedo/who/cf/cf_1007/, consultada el 14.11.2011.

Consejo de Europa (2002). Marco común europeo de referencia para las lenguas. Disponible en: http://cvc.cervantes.es/obref/marco/cvc_mer.pdf, consultada el 14.11.2011.

Gimeno Sanz, A., Martínez Sáez, A., Sevilla Pavón, A., de Siqueira Rocha, J. M. (2011). Fostering autonomy in pedagogically sound e-learning environment, en Sergio Maruenda-Bataller, S. y Clavel-Arroitia, B. (Eds.), Multiple voices in academic and professional discourse: current issues in specialised language research, teaching and new technologies for learners of English for specific purposes. Newcastle upon Tyne: Cambridge Scholars Publishing, 547-560.

Hall, J., Cheng, A., Carlson, M (2006). Reconceptualizing multicompetence as a theory of language knowledge. Applied Linguistics, 27 (2): 220-240.

Gimeno Sanz, A. (2006). Proyecto InGenio: gestor de recursos para el aprendizaje de idiomas. Revista de Didáctica MarcoELE, 2: 1-15.

Meskill, C. y Sadykova, G. (2011). Introducing EFL faculty to online instructional conversations. ReCall, 23 (3): 200-217.

Sevilla, A. y Martínez, A. (2010). El uso de materiales didácticos online destinados a la preparación del nuevo examen informatizado CBFCE. Revista Latinoamericana de Tecnología Educativa, 9 (2): 139-151.

University of Cambridge ESOL Examinations (UCLES), Cambridge ESOL, http://www.cambridgeesol.org/index.html, consultada el 14.11.2011.

Warschauer, M. (2006). Foreword. En Arnó Macià, E., Soler Cervera, A., y Rueda Ramos, C. (Eds.) Information technology in languages for specific purposes: issues and prospects. New York: Springer, xiii-xiv.

\footnotetext{
Referencias de la autora

Ana Sevilla Pavón es licenciada en Filología inglesa y francesa por la Universitat de València. Realiza su tesis doctoral en el Departamento de Lingüística Aplicada de la Universitat Politècnica de València, y es beneficiaria de una beca FPI otorgada por la Generalitat Valenciana. Su actividad investigadora, que realiza en el grupo de investigación CAMILLE - dirigido por Ana Gimeno-, se centra en el diseño de un programa preparatorio y de evaluación online para la realización de exámenes oficiales, dentro del campo de la enseñanza y evaluación asistidas por ordenador. Así, colabora en proyectos de I+D para el estudio de los procesos de cambio y automatización de las Pruebas de Acceso a la Universidad a partir del desarrollo de herramientas online, financiados por el Ministerio de Ciencia e Innovación y por la
} 
Generalitat Valenciana. Ha participado, asimismo, en numerosos congresos de lingüística aplicada y de tecnología educativa, y publicado diversos artículos, capítulos de libro y reseñas.

Email: ansepa@upvnet.upv.es

Para citar este artículo:

Sevilla Pavón, A. (2012). El desarrollo de un programa de evaluación online para la preparación de la versión informatizada del First Certificate in English. Bellaterra Journal of Teaching \& Learning

Language \& Literature, 5(1), 23-38. 